\title{
Automatic elaborative encoding in children's associative memory
}

\author{
DANIEL W. KEE \\ California State University, Fullerton, California 92634 \\ and \\ SUSAN Y. NAKAYAMA \\ University of Southern California, Los Angeles, California 90007
}

\begin{abstract}
The present study was conducted to evaluate pictorial elaboration effects in children's incidental paired associate memory. The design of the experiment consisted of a 2 by 2 by 2 by 5 factorial with grade level (kindergarten vs. second), pictorial presentation (standard vs. elaborated), list (two 14-pair lists of common noun referents), and instruction (a memory control and four different incidental memory conditions). One hundred and twenty children at each grade level were tested individually by the study-test paired associate method. The results indicate that pictorial elaboration facilitated paired associate performance relative to standard presentation; this outcome was not influenced by the factor of age or of instruction. The results were interpreted in terms of an automatic encoding hypothesis.
\end{abstract}

Pictorial elaboration is consistently associated with improved performance relative to standard presentation in children's paired associate retention of nouns (Kee, 1976; Kee \& Rohwer, 1973, 1974). Pictorial elaboration consists of presenting the referents of the to-beremembered (TBR) nouns engaged in a spatial interaction (e.g., a picture of a chain inside a bowl); standard presentation consists of presenting the referents of the TBR nouns side by side (e.g., a picture of a chain next to a bowl).

Typically, pictorial elaboration effects in children's paired associate retention have been evaluated under intentional memory conditions, in which the subject is informed about the pairwise arrangement of TBR referents and requested to study the pairs so that when one member of a pair is presented, the second can be recalled or recognized. A study by Kee and White (1977) assessed the role of memory instruction (inspection vs. memory) on the performance facilitation associated with pictorial elaboration by testing both kindergarten and second-grade children. They found that pictorial elaboration facilitates paired associate performance regardless of whether the child is instructed to remember. Furthermore, while the memory instruction enhanced paired associate retention of the noun referents presented under standard presentation, no difference was observed

This research was supported by Biomedical Science Support Grant RR07012 from the Division of Research Resources, Bureau of Health Professions, Education, and Manpower Training, National Institutes of Health. Appreciation is expressed to the children and staff of the participating schools from the Los Angeles Unified School District for their assistance in the completion of this project. Requests for reprints should be sent to Daniel W. Kee, Department of Psychology, California State University, Fullerton, California 92634. between the instructional conditions for the elaborated presentation of referents. The results also indicated that neither of these forenamed outcomes interacted with the factor of grade. Kee and White advanced an automatic encoding hypothesis to account for these findings. This hypothesis, which is consistent with current research and theory in memory that holds that the memory trace (nature and strength) is an automatic by-product of the kind of cognitive analysis performed on the TBR stimuli (cf. Craik \& Lockhart, 1972; Craik \& Tulving, 1975; Geis \& Hall, 1976), suggests that the unique depiction of the referents under elaborated presentation prompts the child to analyze the shared relational features of the TBR pair members. This analysis, in turn, induces the encoding of the pair members within a common relational episode, thereby enhancing pair-member availability relative to pairs encoded under standard presentation. Important features of this hypothesis are (1) that the intention to relationally encode pair members is not necessary for elaborative coding and (2) that the simple inspection of the TBR referents under elaborated presentation is considered a sufficient condition for the induction of elaborative coding, which facilitates paired associate performance.

The present study was conducted to evaluate the generality of the findings reported by Kee and White (1977) of pictorial elaborative facilitation in incidental paired associate memory. In the Kee and White study, elaborative facilitation was observed under only one type of incidental memory condition. In this condition, the subjects were asked to inspect the referents carefully on the study trial. In addition, the subjects were led to believe that their opinions concerning the referent pairs would be sought after they were presented. This 
disguise, which was used to maintain the credibility of the incidental memory task, may have elicited differential processing of the TBR pair members under standard and elaborated presentation. For example, telling subjects that their opinions would be solicited might have prompted the analysis of the relationship depicted for the pair members under pictorial elaboration, thereby inducing elaborative coding. Since the automatic encoding hypothesis advanced by Kee and White proposes that the mere inspection of referents depicted under elaborated presentation should be sufficient to induce elaborative coding, provisions were made in the present study to assess the pictorial elaboration effect under an inspection condition in which the subjects were asked only to look at the referent pairs (i.e., a pure inspection condition).

In order to exercise some control over the kinds of cognitive activity that might be elicited by the inspection instruction, two additional incidental memory conditions were included in the present study. In each, the children were required to make a well-defined orienting response to the presented pairs. One of these orienting task conditions required the children to express their like or dislike for the way each pair member was drawn. This orienting task should prompt the processing of each member in the pair as an independent entity. This type of processing was expected to be antagonistic to the efficient relational encoding of pair members (cf. Bower, 1970; Dempster \& Rohwer, 1974), thereby providing a robust evaluation of the automatic encoding hypothesis. The other orienting task condition required the children to make a judgment concerning whether or not both members of the pair had been and would be seen together. This orienting task was designed to prompt the child to analyze the shared relational features of the pair members; it provides an opportunity to evaluate the degree to which pictorial elaboration effects are influenced by active relational processing.

Thus, the present study was conducted to provide a refined appraisal of pictorial elaboration effects in children's incidental paired associate memory. Sampling was conducted at the kindergarten and second-grade levels. These age groups correspond to those sampled in the Kee and White (1977) study and provide an opportunity to determine if pictorial elaborative facilitation in incidental paired associate memory varies as a function of development.

\section{METHOD}

\section{Design}

The design of the experiment consisted of a 2 by 2 by 2 by 5 factorial, with grade (kindergarten vs. second), pictorial presentation (standard vs. elaborated), list (two different paired associate lists), and instruction (a memory control and four different incidental memory conditions) as between-subjects factors.

The instructional conditions were as follows. (1) MemoryThe child was told to learn each pair in such a way as to be able to produce the name of one pair member when presented the other on a memory test. The experimenter verbally labeled each referent in the pair on the study trial. (2) Kee and White Inspection-The child was told to inspect the pairs carefully and was informed that his or her opinion of the pictures would be solicited after their presentation. The experimenter verbally labeled each referent in the pair on the study trial. This condition, in combination with the forenamed memory control, permits a replication of the Kee and White (1977) design. (3) Pure Inspection-The child was told to simply inspect each referent pair carefully. No other information was provided, except that the experimenter verbally labeled each referent within the pair on the study trial. (4) Separated Orienting Task-The child was asked two questions about how the pictures were drawn: "Do you like the way the [name of one of the referents] is drawn?" and "Do you like the way the [name of the other referent] is drawn?" This orienting task condition was designed to minimize relational encoding by requiring the subject to analyze each member within the pair as a distinct entity. (5) Conjoined Orienting Task-The child answered two questions about the juxtaposition of the referents within the pair: "Have you ever seen a [name of one referent] and a [name of the other referent] together before?" and "Do you think you will ever see them together again?" Two questions were asked in this condition in order to equate the number of questions given in the two orienting task conditions. This last condition was designed to prompt the subject to process the shared relational features of the TBR pair members; hence, task performance facilitation was expected relative to the memory control condition.

\section{Materials and Procedure}

The materials consisted of two 14-pair lists of black-on-white drawings of common objects that had previously been used by Kee and White (1977). All of the objects were highly familiar to the children (e.g., candle, ship, iron, etc.), and pair members did not share obvious preexperimental associations. Standard pictorial presentation consisted of the depiction of the objects side by side, and elaborated pictorial presentation consisted of the depiction of the objects engaged in a spatial interaction. Two random orders of each list were prepared, one for the study trial and one for the test trial. The line drawings were mounted on $12.7 \times 17.8 \mathrm{~cm}$ cards and presented to the subjects manually.

Children were tested individually in a quiet room at the participating school. Each child was seated at a table to the right of the female experimenter and was given task direction in accordance with the instructional condition. The TBR referent pairs were presented manually at a $10-\mathrm{sec} /$ pair rate on the study trial. Approximately $45 \mathrm{sec}$ after this trial, a single cued recall test was administered. This test consisted of presenting the child one member from each pair and requiring the child to recall the associate. Stimulus cue presentation was both pictorial and auralverbal. The test trial rate was $6 \mathrm{sec} /$ item. At the conclusion of the test, children in each of the incidental conditions were asked if they had expected the memory test. Eighteen children who had anticipated the test were replaced.

\section{Subjects}

One hundred and twenty kindergarten children with a mean age of 5.88 years $(\mathrm{SD}=.32$, range $=5.33-7.00)$ and 120 secondgrade children with a mean age of 7.79 years $(S D=.46$, range $=$ 6.67-10.08) participated in the study. Subjects were drawn from three public elementary schools located in a middle-class suburb of Los Angeles. Subjects within each grade were randomly assigned in equal numbers to 1 of the 20 experimental conditions.

\section{RESULTS}

The dependent variable was the total number of correct responses given on the test trial. The Type I error rate for all tests was set at .05 . Table 1 presents the 
Table 1

Mean Number of Correct Responses as a Function of Grade, Pictorial Presentation, and Instruction

\begin{tabular}{lcccccc}
\hline & \multicolumn{5}{c}{ Instruction } \\
\cline { 2 - 5 } $\begin{array}{l}\text { Pictorial } \\
\text { Presentation }\end{array}$ & $\mathrm{M}$ & $\mathrm{KW}$ & $\mathrm{PI}$ & $\mathrm{S}$ & $\mathrm{C}$ & Mean \\
\hline & \multicolumn{5}{c}{ Kindergarten } \\
Standard & 2.83 & 1.67 & 3.00 & 1.50 & 4.17 & 2.63 \\
Elaborated & 5.83 & 5.92 & 5.58 & 4.25 & 6.67 & 5.65 \\
Mean & 4.33 & 3.80 & 4.29 & 2.88 & 5.42 & \\
& & \multicolumn{5}{c}{ Second Grade } \\
Standard & 2.50 & 2.83 & .92 & .75 & 6.75 & 2.75 \\
Elaborated & 6.33 & 6.50 & 5.83 & 5.25 & 10.42 & 6.87 \\
Mean & 4.42 & 4.67 & 3.38 & 3.00 & 8.59 \\
\hline
\end{tabular}

Note-MSe $(200)=7.10 . \quad M=$ memory, $K W=$ Kee and White inspection, $P I=$ pure inspection; $S=$ separated, $C=$ conjoined .

means for the experimental conditions collapsed over the factor of list.

An analysis of variance indicated that the factors of grade and list were not associated with significant effects $[F(1,200)=3.76$ and $F(1,200)=1.24$, respectively; ps > .05]. A main effect for pictorial presentation was observed: It was found that pictorial elaboration facilitated paired associate performance relative to standard presentation $[\mathrm{F}(1,200)=107.48, \mathrm{p}<.01]$. A Pictorial Presentation by List interaction was also observed $[F(1,200)=4.97, p<.05]$. The form of this interaction indicated that the degree of facilitation associated with pictorial elaboration varied across the two paired associate lists (List 1: standard mean $=3.27$, elaborated mean $=6.07$; List 2 : standard mean $=2.12$, elaborated mean $=6.45$ ). No other interactions with the factor of pictorial presentation were observed. The absence of the Pictorial Presentation by Instruction interaction $(F<1)$ indicates that the magnitude of facilitation associated with pictorial elaboration did not vary across the instructional conditions. Furthermore, the absence of the Pictorial Presentation by Instruction by Grade interaction $(F<1)$ indicates that the forenamed outcome was not affected by grade level.

Only two other sources of significant effect were detected in the overall analysis of variance: a main effect of instruction $[F(4,200)=15.58, p<.01]$ and an interaction between the factors of instruction and grade $[F(4,200)=3.99, p<.01]$. Pairwise comparisons between the instructional conditions were conducted by the Scheffe method within each grade level in order to explicate the Instruction by Grade interaction $(\alpha=.05)$. At the kindergarten grade level, the conjoined orienting task facilitated performance relative to the separated orienting task condition. No other pairwise comparison was significant for this grade level. Within the second grade, the conjoined orienting task condition facilitated performance relative to each of the other instructional conditions, which did not differ from each other.

\section{DISCUSSION}

The results of the present study demonstrate pictorial elaborative facilitation in children's paired associate retention of noun referents across a variety of different incidental memory conditions. The elaborative facilitation observed under the pure inspection condition is consonant with the automatic encoding hypothesis, which proposed that the mere inspection of the referents depicted under pictorial elaboration would be sufficient to induce elaborative coding and the associated performance benefits. Furthermore, the finding of elaborative facilitation under the separated orienting task condition indicates that automatic elaborative encoding can occur under conditions designed to be antagonistic to efficient relational processing.

In the present study, comparable levels of paired associate performance were observed for pictorial elaboration under the memory condition relative to both the pure inspection and the separated orienting task conditions. This finding suggests that automatic encoding may account for the pictorial elaborative facilitation typically observed in studies of intentional paired associate memory in the kindergarten to second-grade age range. Research indicates that older children (e.g., adolescents) have a much greater propensity than young children to spontaneously elaborate or record TBR pair members in intentional paired associate tasks (e.g., Rohwer \& Bean, 1973). Thus, the performance level associated with pictorial elaboration in older children may not always reflect pure automatic processing. That is, older children may augment the automatic encoding of pictorial elaboration with forms of "optional" encoding (cf. Geis \& Hall, 1976), which may serve to enhance the strength of the memory trace for the TBR pairs. Evidence from the present study offers support for this proposition by demonstrating that the strength of the memory trace associated with automatic elaborative coding can be augmented by active relational processing. Recall that at the second-grade level, the conjoined orienting task condition facilitated the retention of both standard and elaborated pairs relative to the other instructional conditions, which did not differ from each other.

At both the kindergarten and second-grade levels, the conjoined orienting task condition facilitated paired associate retention relative to the separated orienting task condition. One implication of this finding concerns the levels-of-processing framework (cf. Craik \& Lockhart, 1972) in memory research, which holds that the memory trace is an automatic by-product of the cognitive operations performed. This finding clearly indicates that in the absence of the intention to remember, a relational analysis establishes a more durable memory trace for the pair members than a separated analysis establishes in young children. Furthermore, this outcome is consistent with previous findings with older children (Dempster \& Rohwer, 1974) and adults (Bower, 1970), which indicate that relational processing of TBR pair members is associated with superior intentional paired associate retention relative to separated processing of TBR pair members.

It will be recalled that Kee and White (1977) found that memory instruction improved paired associate performance relative to the inspection condition for the standard presentation of pairs. This finding, which Kee and White tentatively attributed to the elicitation of rote rehearsal by the memory instruction (Rohwer \& Bean, 1973), was not replicated in this study. Methodological variations between the studies may account for the different findings. Kee and White provided subjects with two consecutive study trials with a 4 -sec/pair presentation rate prior to the cued recall test. In addition, subjects were reminded to study the pairs in preparation for the memory test at the beginning of each study trial. In the present study, subjects were given only one study trial with a 10 -sec/pair presentation rate prior to the cued recall test. The distributed practice coupled 
with the dual memory prompt associated with the Kee and White procedure may have offered a more hospitable environment for the elicitation and sustention of rehearsal relative to the study trial procedures used in the present study.

In summary, the results of the present study indicate that the pictorial elaboration effect can be observed across a variety of incidental paired associate task conditions. Furthermore, the degree of elaborative facilitation observed under the different incidental conditions did not differ from that observed under memory instruction. These outcomes suggest that for kindergarten and second-grade children, elaborative coding of pair members can be automatically induced by the inspection of pictorial elaborations and thus facilitates paired associate performance.

\section{REFERENCES}

Bower, G. H. Imagery as a relational organizer in associative learning. Journal of Verbal Learning and Verbal Behavior, 1970, 9, 529-533.

Craik, F. I. M., \& Lockhart, R. S. Levels of processing: A framework for memory research. Journal of Verbal Learning and Verbal Behavior, 1972, 11, 671-684.

Craik, F. I. M., \& Tulving, E. Depth of processing and the retention of words in episodic memory. Journal of Experimental Psychology: General, 1975, 104, 268-294.
Dempster, F. N., \& Rohwer, W. D., Jr. Component analysis of the elaborative encoding effect in paired-associate learning. Journal of Experimental Psychology, 1974, 103, 400-408.

GEIS, M. F., \& HALL, D. M. Encoding and incidental memory in children. Journal of Experimental Child Psychology, 1976, 22, 58-66.

KEE, D. W. Storage and retrieval of noun-pairs in children's recognition memory: Analysis of presentation mode and elaboration effects. Journal of Experimental Psychology: Human Learning and Memory, 1976, 2, 623-632.

KeE, D. W., \& Rohwer, W. D., JR. Noun-pair learning in four ethnic groups: Conditions of presentation and response. Journal of Educational Psychology, 1973, 65, 226-232.

KeE, D. W., \& Rohwer, W. D., JR. Response and associative phase components of elaborative prompt effects in children's noun pair learning. Journal of Experimental Child Psychology, $1974,18,62-71$.

KeE, D. W., \& White, B. R. Children's noun-pair learning: Analysis of pictorial elaboration and memory instruction effects. Child Development, 1977, 48, 674-677.

RoHWER, W. D., JR., \& BEAN, J. Sentence effects and noun-pair learning: A developmental interaction during adolescence. Journal of Experimental Child Psychology, 1973, 15, 521-533.

(Received for publication September 3, 1980.) 